\title{
EFECTO DE LA EDUCACIÓN MUSICAL AL DESARROLLO INTEGRAL DE LOS NIÑOS EN LA ETAPA PRE-ESCOLAR
}

\author{
Carrión Diego ${ }^{1}$, Cabrera Julio ${ }^{2}$. Carmen Aguirre ${ }^{3}$ \\ diegocarrion@mail.ru',judicafi@gmail.com² \\ https://orcid.org/0000-0002-5433-5266 ${ }^{1}$, https://orcid.org/0000-0002-7239-5780 ${ }^{2}$ \\ Universidad estatal Rusa Kosygina ${ }^{1}$ \\ Facultad Instituto de música Academia Maimonides \\ Moscú - Rusia
}

Recibido (01/07/20), Aceptado (17/07/20)

\begin{abstract}
Resumen: Este artículo presenta los resultados de un trabajo de investigación, sobre los efectos de la educación musical y su incidencia en el desarrollo integral de los niños de la etapa pre-escolar, el cual se lo ha llevado a su final. A través de dicha investigación se promueve la utilización a la música como eje trasversal para el proceso de aprendizaje en niños del Ecuador del sector rural, de una parroquia dentro del cantón Quito. El desarrollo metodológico se ha basado en una investigación de campo y enfoques teóricos, con los que se sustentan la investigación. Se puede mencionar que al aplicar la música en la etapa pre-escolar ayudara a desarrollar el proceso mental mediante la estimulación en ambientes significativos, los cuales permitirán un aprendizaje duradero
\end{abstract}

Palabras Clave: Educación musical, desarrollo integral, educación inicial

\section{EFFECT OF MUSIC EDUCATION ON THE COMPREHENSIVE DEVELOPMENT OF CHILDREN IN THE PRESCHOOL STAGE}

Abstract: This article presents the results of a research work on the effects of music education and its impact on the comprehensive development of children in the pre-school stage, which has led to its end. Through this research, the use of music is promoted as a transversal axis for the learning process in children from Ecuador in the rural sector, from a parish within the canton of Quito. The methodological development has been based on field research and theoretical approaches, with which the research is sustained. It can be mentioned that applying music in early childhood will help develop the mental process by stimulating in significant environments, which will allow lasting learning.

Keywords: Music education, integral development, initial education 


\section{I.INTRODUCCIÓN}

Es importante considerar que el iniciar de manera temprana la enseñanza de la música favorecerá los distintos aprendizajes tanto cognitivos, afectivos y psicomotrices [1]. Sin embargo, la música no ha sido relacionada en tal dimensión a pesar de que algunos estudios consideran que la música es el mejor recurso para la educación. Si retomamos un poco de historia se puede repasar como aparece la música en Estados Unidos de Norteamérica en las escuelas públicas [2]. Poco a poco esta asignatura va incorporándose, hasta que en 1860 la mayoría de instituciones la adopta en sus programas de estudio [2]. Desde esta perspectiva la educación musical en Latinoamérica ha estado tratándose de implementar con esfuerzo desde 1941 con la Unión Panamericana, considerando que el estado americano siempre tuvo un gran interés por el desarrollo musical, en lo profesional como en lo educativo. Por diez años no tuvieron éxito, y cambiaron la estrategia, delegando así a cada uno de los maestros para que incentiven y promuevan la importancia de la educación musical a sus gobiernos [3]. Problema que lo evidenciamos hasta la actualidad el arte y en sí la música son excluidas o tratadas como materias opcionales, sin ningún valor, cuando en la realidad ésta podría ser utilizada para potenciar todo el sistema del desarrollo integral de los niños. El arte siempre será visto como gasto y no como inversión.

A la suma de esto los niños de la zona rural en donde se realiza la investigación, no tienen todo lo necesario para poder educarse, siempre faltan profesores, $\mathrm{y}$ no todos los maestros tienen el conocimiento en educación musical, el entorno familiar es otro factor que influyen pues se desenvuelven en hogares mayormente conflictivos e inestables que les obliga a alejarse de las emociones positivas, sumergiéndose en esferas de agresión, violencia registrando como consecuencia de esta inestabilidad, los altos índices de alcoholismo, hogares disfuncionales, pobreza, desempleo.

La presente investigación trata de puntualizar como la educación musical incide en el desarrollo integral de los ninos, y como debe ser tratada como un recurso didáctico para desarrollar actitudes y aptitudes en los estudiantes.

La recolección de la información se realizó mediante una encuesta aplicada a 3 docentes, lista de cotejo a 30 niños de la Escuela Fiscal Mixta Julio María Matovelle, de tal manera nos reflejó datos importantes para poder realizar el trabajo de investigación.

Este trabajo se detalla de la siguiente manera: La importancia de la educación musical y la relación con el desarrollo integral del niño, en la segunda sección encontramos la problemática de investigación, y en la tercera se evidencia la utilización de la metodología y la última sección presentaremos los resultados.

\section{II.DESARROLLO}

La educación musical a través de la historia permite considerar como aparece en nuestro continente por primera vez, implantada en estados unidos casi a mediados del siglo XIX 1838, en todas las escuelas de Boston, y tan solo 20 años después implementada en todo el país [2].

Y al pasar del tiempo en el siglo XX aparece como una asignatura especial y su término se lo reconoce por primera vez en Estados Unidos [2]. En este tiempo para fortalecer el estudio de la música se fundó la conferencia nacional de Educadores en Música, los cuales realizaron sus trabajos de investigación, material didáctico, redactando directrices, material de información, talleres, capacitando para obtener los mejores resultados, y así poder difundirlo al mundo entero.

En los países de América Latina, también se implementaron, en algunos casos se lo aplicaron de mejor manera y otros con limitaciones, pero llegó un punto de olvido, y aunque en 1941 la Organización de los Estados Americanos se esforzó para difundir la educación musical, no logró recibir los resultados que esperaba, por ello cambiaron sus estrategias porque entendieron que tratar de cambiar las cosas desde afuera hacia adentro no funcionaba, pues llegaron con sus propuestas, a querer implantar sin saber la realidad social de cada país y su problemática, pue fue así como decidieron implantar talleres de capacitación para los maestros, porque de nada serviría las buenas propuestas si los maestros de música no están capacitados, no podrían poner en marcha ningún plan . Gracias a un visionario el Dr. Ernesto Rizo rector del conservatorio Nacional de Nicaragua, aseguro todo el apoyo del gobierno para llevar a cabo esta idea, y es así como la Universidad del Rosario en Nicaragua se convirtió en la sede del primer curso centroamericano de Educación musical en 1971 [2].

\section{A.Desarrollo Integral del Niño.}

En breves rasgos se ha podido evidenciar como la música a través del tiempo va tomando su importancia, es así como se la debe involucrar en todas las áreas de educación en los distintos niveles. De manera indispensable en los primeros años de vida del niño, es decir en el nivel inicial, puesto que en estas edades se desarrollan los campos cognitivos mediante experiencias significativas, y es la música la idónea para permitir la expresión, sensibilidad, imaginación y comunicación de sentimientos [4].

Otro de los aspectos que ayuda a desarrollar la músi- 
ca es el área afectiva y por ende el desarrollo social en el cual influencia su entorno involucrado los estímulos con actividades musicales las mismas que producirán cambios neurológicos en el cerebro [5]. Abordando de esta manera la memoria de percepción sonora, aspectos bilógicos de la conducta musical y las funciones de la corteza auditiva [5].

\section{B.Primeros rasgo de musicalidad.}

La música está presente en el desarrollo del niño desde su primera manifestación por medio del llanto, puesto que tiene cualidades expresivas sonora por las cuales el infante se comunica, el lloro puede expresar una necesidad como hambre, sueño, cansancio, la conciencia musical aparecerá después del año, el niño estará listo para notar la relación secuencial entre diferentes sonidos [5].

Sin embargo las primeras manifestaciones musicales en el niño es el balbuceo puesto que están compuestos por glisandos microtonales con suaves movimientos reconociendo tonos familiares discriminando así sonidos musicales de los no musicales [5].

En la etapa preescolar actualmente reconocido como nivel inicial, se desarrolla el canto espontaneo, a esta edad empiezan a imitar sonidos de su entorno, y lo primero que imitan son palabra no completas, luego pasan a la repetición de canciones es decir, empieza con la imitación de palabras, luego fragmentos melódicos y posteriormente canciones completas [5].

Es indispensable mencionar que las habilidades musicales en los primeros años se desarrollaran en un ambiente sonoro [5].

Si hablamos de los aspectos que la música ayuda a progresar, se puede apreciar la influencia que tiene en el desarrollo integral, puesto que ayuda al desenvolvimiento del área cognitiva, afectiva y psicomotriz [1]. Por otro lado, al estimular la inteligencia musical se activará el razonamiento lingüístico y lógico matemático, implicando la participación del hemisferio dominante [1]. El niño en esta edad inicial tiene un aprendizaje por imitación.

\section{C.Influencia del Entorno.}

El rol del docente y la familia es primordial en este proceso de estimulación musical, en este aspecto se hablará primero sobre el docente pues quien tiene que ser un facilitador que proporcione experiencias significativas, las mismas que se enfoquen en los intereses de esta edad inicial [6].

El contexto familiar debe proporcionar condiciones que faciliten el avance del ser humano suministrando ambientes armónicos [7].
En este estudio es indispensable proponer a la cultura como parte del entorno del niño, pues mediante esta se puede comunicar emociones, pensamientos, declaraciones políticas, relaciones sociales y expresiones física todas estas manifestaciones declaran una identidad, valores, creencias que se manifestadas en las costumbres de cada comunidad esto se puede comprobar en las instituciones que se encuentran en sectores rurales [8].

\section{III.METODOLOGÍA}

La metodología de este trabajo es probabilístico intencional, está formada por 3 docentes y 30 niños de la Escuela Fiscal Mixta Julio María Matovelle, en la comuna de San Antonio de Cucupúro a $5 \mathrm{Km}$ de la parroquia de El Quinche a más de $3000 \mathrm{msm}$, en el cantón Quito, Provincia de Pichincha Ecuador.

El instrumento utilizado fue una encuesta descriptiva, dicotómica y de selección, basada en un conjunto de preguntas de 12 ítems, de las cuales 5 preguntas están en la escala de tipo Likert de 4 a 8 alternativas de respuesta. La encuesta está estructurada por 3 componentes. El primer componente: Está relacionado con la educación musical; El segundo: corresponde al proceso de enseñanza aprendizaje; y el tercer elemento es: Sobre el desarrollo integral. Por otro lado, la investigación contó con una lista de cotejo para evaluar el desarrollo integral de los niños.

La recolección de la información se realizó con la permisión de la autoridad de la institución educativa para la aplicación de los instrumentos de investigación para los docentes y estudiantes. A los maestros se les informó el objetivo de la investigación, y el manejo responsable de los datos obtenidos.

\section{IV.RESULTADOS}

Como se había expuesto en la primera parte, la importancia de aplicar la educación musical para su incidencia en el desarrollo integral del niño. Los resultados de la encuesta, arrojaron datos favorables y las maestras consideran que es importante la educación musical como eje trasversal en el desarrollo del niño.

Al observar los resultados favorables en la encuesta se evidencia que, al aplicar la educación musical en el niño, le ayuda a desarrollar a ser más sociables, analíticos y autónomos.

Al obtener la información para la realización de las tablas, podemos comparar los resultados. Aquí se presentan algunas de las preguntas presentadas en la encuesta que proyectaron efectos significativos. 
TABLA I. Aplicación de los elementos fundamentales del área de lenguaje y expresión musical.

\begin{tabular}{|c|c|c|}
\hline Indicadores & Frecuencia & Porcentaje \\
\hline Canción & 3 & $100 \%$ \\
\hline Audición & 0 & $0 \%$ \\
\hline Danza & 0 & $0 \%$ \\
\hline Ritmo & 0 & $0 \%$ \\
\hline TOTAL & 3 & $100 \%$ \\
\hline
\end{tabular}

Fuente: Encuesta aplicada a docentes de Educación Inicial de la Escuela Fiscal Mixta Julio María Matovelle del Quinche, cantón Quito, provincia de Pichincha.

Responsable: Los Autores.

Los resultados determinan que todas las maestras recurren a las canciones porque en ellas pueden encontrar varios elementos didácticos de la música tanto para comprensión como para la expresión musical. El tratamiento de la voz encuentra en la canción su principal marco de desarrollo. La canción se puede apoyar con gestos, palmadas y movimientos. Los cantos grupales posibilitarán la coordinación con el grupo de iguales, el volumen y el ritmo en conjunto, el acompañamiento con instrumentos sencillos. El canto es uno de los medios de expresión más completo y máximo de la actividad musical, que lleva al ser humano a descubrir la imagen del propio cuerpo.

En otros análisis de respuestas dicotómicas los resultados justifican la importancia de la preparación de la estimulación musical, consideran que la mayoría de actividades deben estar basados en fundamentos psicológicos de música. Expresan que la psicología de la música considera a la persona como un ser integral: cuerpo y mente; emoción y espíritu. Ayuda al desarrollo psíquico y emocional.

La música nos conduce a una armonización de los estados de ánimo y de los sentimientos. Permite un equilibrio dinámico entre las capacidades del hemisferio izquierdo y derecho. Da lugar a un aprendizaje mucho más equilibrado y adaptado tanto al medio, como a las propias capacidades individuales. Dentro de esta individualidad, junto a la complejidad cerebral, consideramos a la música como uno de los elementos con mayor capacidad para la integración neurofuncional y neuropsicológica. Contribuye a desarrollar la percepción sonora, estados de ánimo, conductas cognitivas, perceptivo-motrices. La actividad se sintetiza en una función tanto receptiva como ejecutiva del cerebro, que permite modificar conductas.

Por lo tanto, los elementos fundamentales de la música no son tan sólo elementos componentes formales, materiales sino también, ante todo, elementos de vida, propios de todo ser humano.
Las maestras encuestadas afirman que la estimulación musical influye notablemente en el desarrollo integral de los niños. El niño que vive en contacto con la música aprende a convivir de mejor manera con otros niños, estableciendo una comunicación más armoniosa. A esta edad la música les encanta, les da seguridad emocional porque se sienten comprendidos al compartir canciones, e inmersos en un clima de ayuda, colaboración y respeto. La música también ofrece una placentera y provechosa experiencia de aprendizaje y alimenta la imaginación y la creatividad de los niños. Por lo tanto, no se puede prescindir de la música para el desarrollo integral.

El resultado del nivel de la investigación en la lista de cotejo nos reflejó los siguientes porcentajes.

El $40.5 \%$ de los niños se desenvuelven de manera autónomo y tiene un desarrollo integral favorable. El $37.9 \%$ de estudiantes tienen un nivel bajo en su desarrollo general, y el $21.5 \%$ de los niños manifiestan un escaso desarrollo de crecimiento, tanto como perceptivo, lingüístico, físico, mental, emocional y social.

Los resultados enfocados a la educación musical del niño, dan una visión amplia sobre la importancia que tiene en el desarrollo integral, con la aplicación correcta y esmerada podremos tener en un futuro personas equilibradas, colaboradoras y una ser humano dispuesto a transformar a bien la sociedad.

\section{V.CONCLUSIONES}

Los resultados de nuestro trabajo, nos revelan que la música utilizada como herramienta para el desarrollo integral del niño presentó un importante avance en el progreso de la investigación, y los datos obtenidos antes y después nos refleja resultados importantes al beneficio de los educandos.

La música siempre será una herramienta que se la puede combinar en muchas áreas sobre todo dentro de la educación. Empezando aplicar en actividades primitivas del sonido, como son sus cualidades: altura, tim- 
bre, duración, e intensidad.

$\mathrm{Al}$ analizar la influencia de la música en el desarrollo del niño como eje transversal para la educación se observa que su aporte es fundamental en el plano educativo, pues su aprendizaje influye en los procesos cognitivos demostrando y dando la pauta para combinar otros métodos pedagógicos, que estén relacionados en este campo, así se puede dar paso a una nueva investigación en el área lúdica expresiva.

\section{REFERENCIAS}

[1]M. V. Casas, «¿Por qué los niños deben aprender música?,» Red de revistas científicas de américa látina, vol. 32, no 4, pp. 197-204, 2001.

[2]M. L. Muñoz, «La educación musical en latinoamerica,» Revista muscial chilena, vol. 30, no 134, pp. 5668, 1976.

[3]D. M. L. Muñoz, «La Educación Musical en Latinoamérica.,» Revista Musical Chilena, pp. 56-68.

[4]I. X. Galicia ,Moyeda y L. Zarzosa, Escobedo , «La

\section{RESUMEN CURRICULAR}

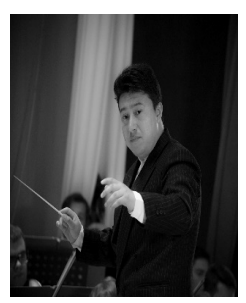

Carrión Granda, Diego Fernando; Nacido en Ecuador, Director de Ópera y Orquesta Sinfónica reside en Moscú-Rusia desde el 2015. Alumno de Vládimir Alexándrovich Pónkin. Fundador y director principal de la Orquesta YOI "Young Integration Orchestra" desde el 2019, y organizador del concurso anual de composición "Luis Gerardo Guevara Viteri". www.diego-carrion.com presencia de las actividades musicales en los programas educativos y su influencia en la conciencia fonológica,» Revista Scielo, vol. 36, n 144, pp. 157-172, 2014.

[5]G. P. Zapata , «Reflexiones desde la educación musical y la psicología de la múscia sobre el desarrollo socio-afectivo y musical de niños de comunidades vunerables,» Revista de la facultad de artes, pensamiento palabra y obra, $n^{\circ} 6$, Julio-Diciembre 2011.

[6]J. E. Mendoza, Carrera, E. G. Montero, Zamora, G. J. Castro, Castillo y D. J. Burgos, Mendieta, «Las artes y su incidencia en todos los niveles educativos de la universidad estatal de milagro,» Revista universidad ciencia y tecnología, vol. 2, nº 2, pp. 63-67, 2019.

[7]R. Perea, Quezada, «La familia como un contexto para un desarrollo saludable,» Revista española de pedagogía, no 235, pp. 417-428, 2006.

[8]A. Porta, «La construcción de la identidad en la infancia y su relación con la música,» Revista de ducación en humanidades, $n^{\circ}$ 5, pp. 61-76, 2014.

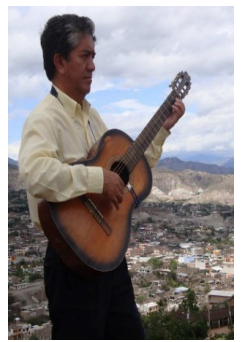

Cabre ra Figueroa, Julio Divar; Docente ecuatoriano, Dr. en Ciencias de la Educación mención investigación educativa, Lic. y profesor en docencia primaria, pedagogía musical. En la actualidad está jubilado del magisterio ecuatoriano con 35 años de servicio, y está desarrollando su propia metodología para la enseñanza del piano. 